\title{
Infants Anticipate Others' Social Preferences
}

\author{
Christine Fawcett ${ }^{\mathrm{a}, *}$ and Ulf Liszkowski $\mathbf{i}^{\mathrm{a}, \mathrm{b}}$ \\ ${ }^{a}$ Max Planck Research Group Communication Before Language, Max Planck \\ Institute for Psycholinguistics, Nijmegen, The Netherlands \\ ${ }^{\mathrm{b}}$ Donders Institute for Brain, Cognition and Behaviour, Radboud University, \\ Nijmegen, The Netherlands
}

In the current eye-tracking study, we explored whether 12-monthold infants can predict others' social preferences. We showed infants scenes in which two characters alternately helped or hindered an agent in his goal of climbing a hill. In a control condition, the two characters moved up and down the hill in identical ways to the helper and hinderer but did not make contact with the agent; thus, they did not cause him to reach or not reach his goal. Following six alternating familiarization trials of helping and hindering interactions (help-hinder condition) or up and down interactions (up-down condition), infants were shown one test trial in which they could visually anticipate the agent approaching one of the two characters. As predicted, infants in the help-hinder condition made significantly more visual anticipations toward the helping than hindering character, suggesting that they predicted the agent to approach the helping character. In contrast, infants revealed no difference in visual anticipations between the up and down characters. The up-down condition served to control for low-level perceptual explanations of the results for the helphinder condition. Thus, together the results reveal that 12month-old infants make predictions about others' behaviour and social preferences from a third-party perspective. Copyright (C) 2012 John Wiley \& Sons, Ltd.

Key words: social cognition; visual anticipation; eye tracking; infant development

\section{INFANTS ANTICIPATE OTHERS' SOCIAL PREFERENCES}

From very early in life, realizing whether others' social actions are positive or negative is a valuable social-cognitive ability. This is true both from a first-person

*Correspondence to: Christine Fawcett, The Uppsala Babylab Department of Psychology Uppsala University, Box 1225, 75142 Uppsala, Sweden. E-mail: christine.fawcett@psyk.uu.se 
perspective-while one is part of the interaction-and from a third-person perspective-while one is observing others interact. In addition, being able to use these social evaluations to predict others' actions is even more beneficial. Using others' past behaviour to predict their future behaviour can allow individuals to make informed decisions about whom to interact with and whom to avoid.

Studies using habituation reveal that 12-month-old infants show passive understanding of positive and negative interactions that they have observed as a third party (e.g. Premack \& Premack, 1997). Twelve- and 10-month-old infants' looking time suggests that they find it more consistent for goal-directed agents to approach those who helped them in the past (Hamlin et al., 2007a, b; Kuhlmeier et al., 2003), and 3- to 10-month-old infants themselves prefer to look at or reach for those who helped another (Hamlin et al., 2007a, b; Hamlin \& Wynn, in press), even compared with a neutral character (Hamlin et al., 2010). However, the question of whether infants also have the ability to predict others' behaviour in such situations, a more stringent test of infants' understanding, has not yet been addressed. These previous studies can only reveal infants' reactions to events after they have occurred, not their perceptions and expectations during an ongoing event. In the domain of object perception, making predictions about objects' motion has been shown to be more challenging than reacting to violations of possible object motions (Hood et al., 2003), and in the social domain, reacting to atypical actions between two people is an earlier development than visually predicting typical actions (Gredebäck \& Melinder, 2010). Being able to predict another's behaviour is essential because it allows one to plan one's own actions in advance of another's behaviour rather than only to respond to another's behaviour after it happens. The current study uses eye tracking to examine 12-month-old infants' behavioural anticipations based on others' social interactions to test whether they possess this more advanced level of understanding about others' prosocial behaviour.

Eye tracking is a valuable tool for studying infants' action predictions using the measure of gaze anticipation (Aslin, 2007; Gredebäck et al., 2010). For example, with repeated viewings, 1-year-old infants gaze ahead to the container where someone will place an item (Falck-Ytter et al., 2006; Gredebäck et al., 2009) or the body part where an object will likely be directed (Gredebäck \& Melinder, 2010; Hunnius \& Bekkering, 2010). In addition, 9-month-old infants have recently also been shown to predict others' locomotion along one path versus another (Paulus et al., 2011).

Using an eye-tracking paradigm, we built on the previous studies by Kuhlmeier, Hamlin, and colleagues to examine whether 12-month-old infants can predict the behaviour of an agent toward those who helped or hindered him in achieving a goal. We compared this to a control condition in which the other characters acted similarly but without contacting the agent. In this case, they did not cause him to reach or not reach his goal. We selected this control condition because it allowed us to manipulate the causal relationship between the characters' movements while retaining maximal similarity between the movements and the animacy of the characters across the two conditions. Research on infants' understanding of motion shows that they can use cues such as contact and basic temporal contingencies to distinguish causal from non-causal movement events within the first year of life (Cohen \& Amsel, 1998; Leslie, 1984; Oakes, 1994). Thus, infants in our study should be able to recognize the difference in causality between the two conditions, leading to different evaluations of the interactions. 
In the current study, infants viewed pairs of events in which an agent was attempting to climb a hill and was alternately helped by one character and hindered by another (help-hinder condition; based on the original stimuli from Kuhlmeier et al., 2003) or alternately accompanied up the hill by one character and down the hill by another with no contact in either case (up-down condition). Thus, the two conditions differed perceptually only in whether the characters' actions included causal contact or not. However, the social interpretation of each is quite different. Helping the agent is a more positive social interaction than hindering him, whereas accompanying the agent up or down the hill are both neutral interactions. We examined which character infants anticipated the agent to approach in order to test whether infants predict an agent's preferences based on his previous interactions. We expected that in the help-hinder condition, infants would predict the agent to approach the previously helpful character, whereas in the up-down condition, they would show equal predictions to both characters.

\section{METHOD}

\section{Participants}

Participants included 63 12-month-old infants (35 female; mean age: 12 months 20 days, standard deviation 8 days) randomly assigned to either the help-hinder $(n=32)$ or up-down $(n=31)$ condition. An additional 20 infants were excluded from the analyses: nine for recording no gaze data during the test trial data, eight for not finishing the study because of fussiness, two for experimenter error and one for parental interference. Infants were recruited from a database of families who expressed interest in participating in research with their children. Infants came from primarily White, middle-class backgrounds and lived in a medium-sized European city or surrounding villages.

\section{Stimuli}

Animated video stimuli depicted the movements of three brightly coloured characters (a red circle, a yellow square and a blue triangle) up and down a threedimensional green hill against a white background.

During the help-hinder familiarization, the agent (circle) attempted to climb a hill. After a failed attempt, he was either helped or hindered by one of the remaining characters (square or triangle). Specifically, the agent first made it part of the way up the hill to the first plateau and then made two attempts to reach the top, each time sliding back down. Then, in the help scenario, the helper (e.g. square) descended from the top of the screen and proceeded to push the agent to the top of the hill by bumping him four times as they ascended. In the hinder scenario, the hinderer (e.g. triangle) descended from the top of the screen and proceeded to push the agent back to the bottom of the hill by bumping him four times as they descended. In the end, the helper/hinderer returned to the top of the screen, and the agent remained in place at the top (help condition) or bottom (hinder condition) of the hill (see Figure 1). The entire sequence of events took $13 \mathrm{~s}$, and the stimuli were controlled for the amount of interaction time and amount of contact between the agent and helper/hinderer. 
Familiarization

Help

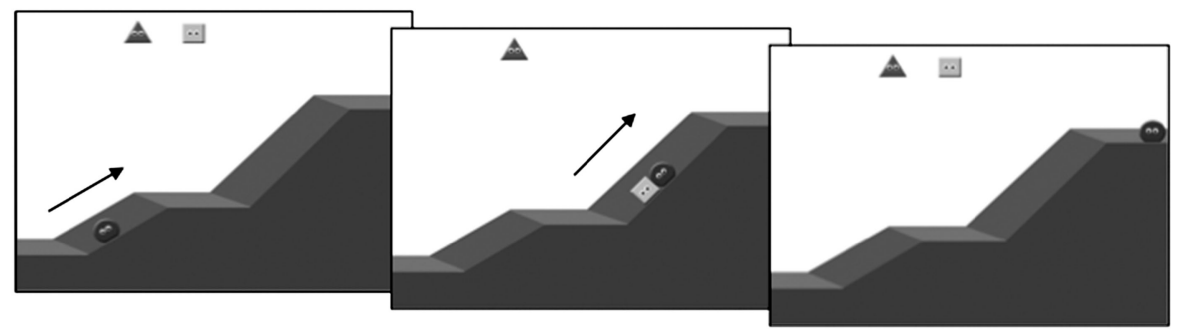

Hinder
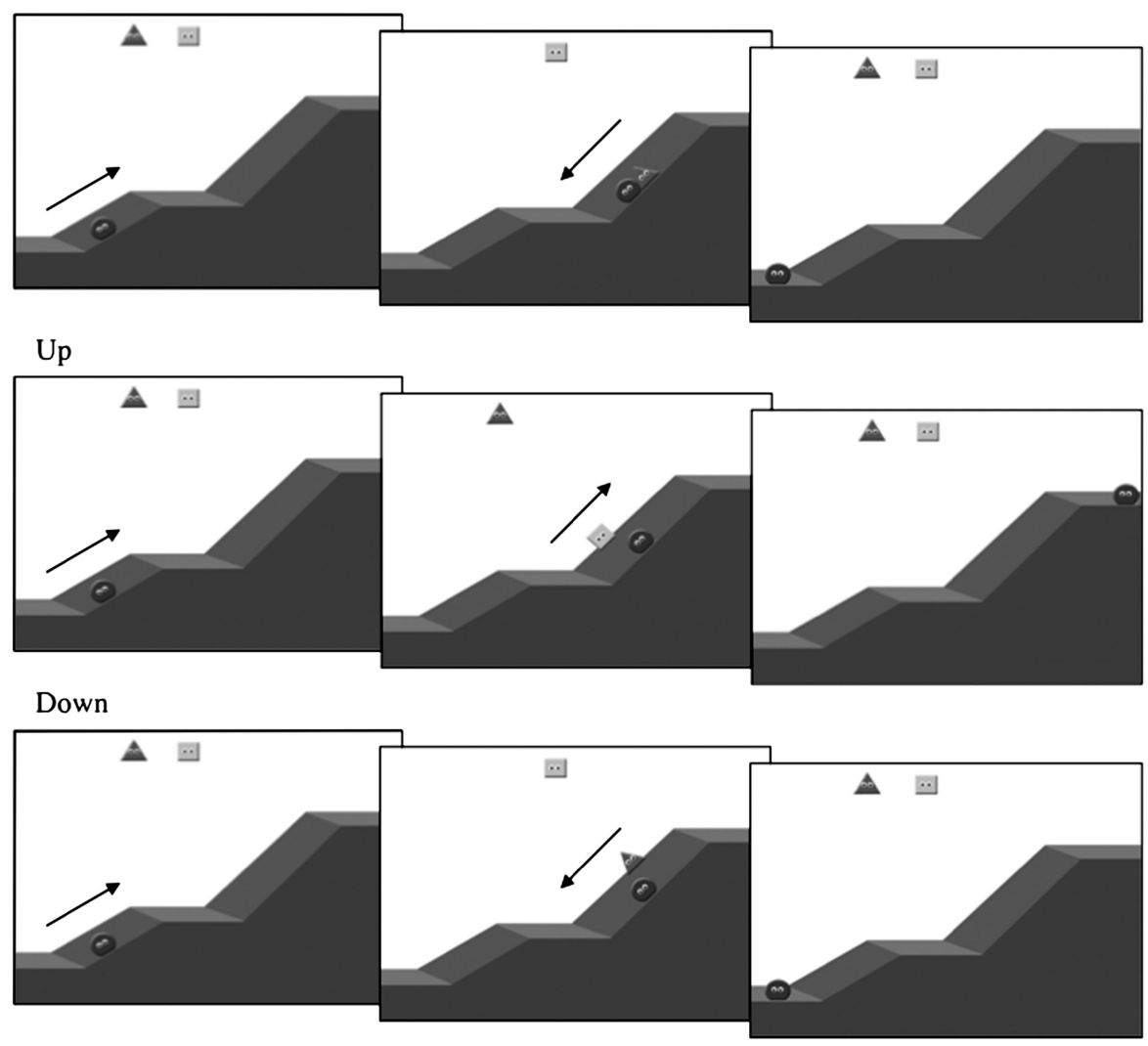

Test

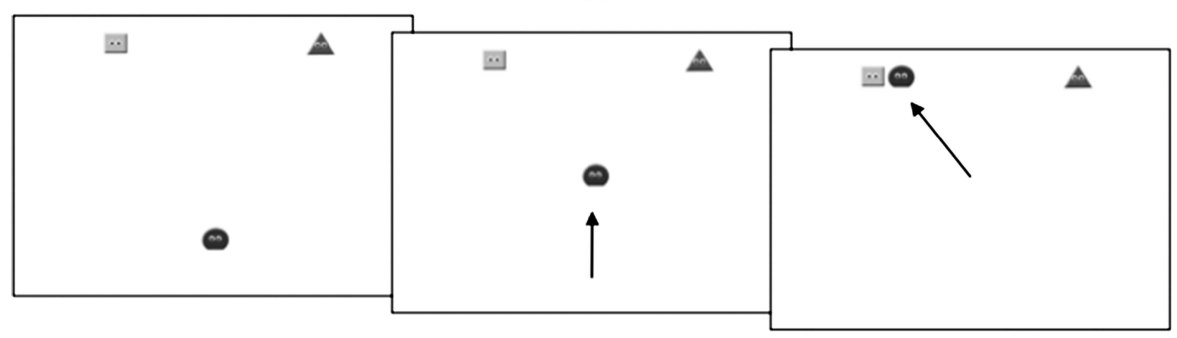

Figure 1. Sequences of actions in the familiarization videos and test video. 
The up-down familiarization was identical to the help-hinder except that the agent and the other character did not come into contact. The same motions, including four short stops and starts (the 'bumping' from the previous condition) were displayed, but the two shapes were separated so that they appeared to be moving next to each other on the three-dimensional hill (see Figure 1). The distance between the agent and the up or down character during their interaction varied slightly with an average of 27 pixels-approximately half the agent's height.

During the test trial, the screen was white except for the helper/up and hinderer/down at the top of the screen and the agent centred at the bottom (see Figure 1). The agent began to approach the helper/up and hinder/down, travelling in a straight line for $1.5 \mathrm{~s}$. He wiggled and then paused, as if deciding which direction to take, for $2 \mathrm{~s}$. Then, he approached either the helper/up or hinderer/down directly for $1.5 \mathrm{~s}$ and finally sat next to the character he approached for $4 \mathrm{~s}$. The entire test sequence took $9 \mathrm{~s}$.

\section{Procedure}

Infants were tested using a Tobii 1750 remote corneal reflection eye tracker (Danderyd Sweden) with infant add-on. The Tobii 1750 has a 50-Hz sample rate, an accuracy of $0.5^{\circ}$ and precision of $1^{\circ}$ and allows head movements of up to about $30 \mathrm{~cm}$ horizontally, $16 \mathrm{~cm}$ vertically and $20 \mathrm{~cm}$ in depth. Infants sat in a car seat that was placed on their parent's lap. They were positioned to sit approximately $50 \mathrm{~cm}$ from the screen. Infants were calibrated using a nine-point calibration before the experiment began.

During the experiment, infants saw a total of six familiarization videos, alternating between help and hinder or up and down, before seeing one test video. The stimuli were also counterbalanced for which shape was the helper versus hinderer or up versus down as well as for which video infants saw first and which character was approached in the test trial. In the final sample for the help-hinder condition, 13 infants saw the square as the helper, and 19 saw the triangle as the helper. In the final sample for the up-down condition, 15 infants saw the square as the up character, and 16 saw the triangle.

\section{Data Reduction}

Areas of interest were created around each of the characters for the test phase (see Figure 2). The critical time phase for infants' anticipation was while the agent approached the two characters in a straight line during the first $3.5 \mathrm{~s}$ of the test trial

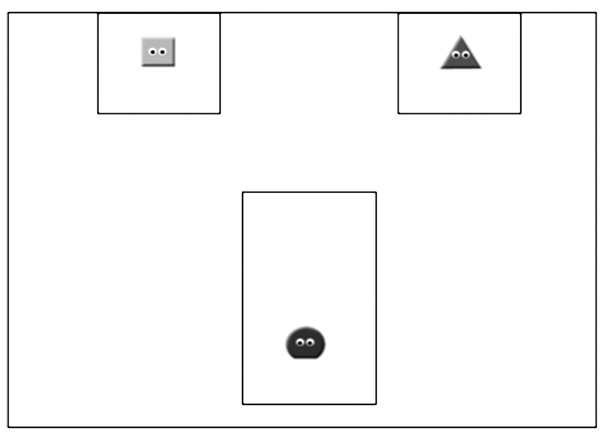

Figure 2. Areas of interest for the test video. 
(anticipation phase). From $3.5 \mathrm{~s}$ until the end of the trial was when the agent had already turned toward one of characters for his final approach and then paused next to that character (approach phase). Gaze data were reduced into fixations in which the infant's gaze remained in an area with a radius of 30 pixels for at least $100 \mathrm{~ms}$.

For the anticipation phase, we required infants to first fixate on the agent such that gaze to the other characters could be considered to indicate where they thought he would approach. Thus, all fixations following infants' first gaze to the agent were totalled, and a proportion of fixation time to the helper or up character was calculated (e.g. duration of fixations to helper/(duration of fixations to helper + duration of fixations to hinderer)). For the approach phase, proportions of looking to the helper or up character were calculated in the same manner but without a requirement to first gaze at the agent.

\section{RESULTS}

Our primary question was whether infants would anticipate the agent's approach to one of the two characters. Thus, we analysed the proportion of infants' gaze directed to each of the two characters, following a fixation on the agent. Approximately half of the infants in each condition did not make any anticipatory gaze, and this rate did not differ across conditions (help-hinder: 17/32, up-down: $\left.15 / 31 ; X^{2}(1)=0.02, p=0.90\right)$. Because the final sample included unequal numbers of infants with the blue triangle versus yellow square as the target, we also included target shape in the analyses to control for this possible confound.

Consistent with our predictions, infants in the help-hinder condition directed significantly more anticipatory gaze to the helper than expected by chance (proportion of gaze to helper $=0.72 ; t(16)=2.58, p=0.02$ ), whereas infants' anticipatory gaze in the up-down condition did not differ across the two characters (proportion of gaze to up $=0.42 ; t(14)=-0.63, p=0.54$; see Table 1 for complete gaze means and standard deviations). A regression analysis confirmed that infants had more anticipatory gaze to the helper (versus hinderer) than to the up (versus down) (condition; $B=0.48, t(28)=2.50, p=0.02$; see Figure 3 ) and that there was no effect of target shape (blue triangle versus yellow square; $B=0.16$, $t(28)=0.84, p=0.41)$ or interaction between shape and condition $(B=0.39$, $t(28)=1.46, p=0.16)$. Infants' first gaze shift following their initial fixation on the agent was also more likely to be directed to the helper rather than hinderer (12 out of 17 infants, binomial $p=0.047$ ), whereas first gaze shifts were at chance in the up-down condition (7 out of 15 infants, binomial $p=0.196)$. Thus, infants do seem to anticipate that the agent will approach the character who helped him over the one who hindered him, but they do not have similar expectations for the agent to approach characters who simply accompanied him up or down the hill.

Once the agent made a decision to approach one character, infants gazed to that character regardless of whether it was the helper, hinderer, up or down. Specifically, during the phase in which the agent approached and paused next to one character, infants tended to gaze more at the approached character than expected by chance (help-hinder: proportion gaze to approached $=0.74, t(30)=4.75, p<0.001$; up-down: proportion gaze to approached $=0.77, t(28)=6.46, p<0.001$; see Table 1 for complete gaze means and standard deviations). A further regression analysis revealed that there was no effect of condition $(B=-0.05, t=-0.47, p=0.64)$ or whether the target character (help or up) was approached $(B=-0.28, t=-1.45$, 


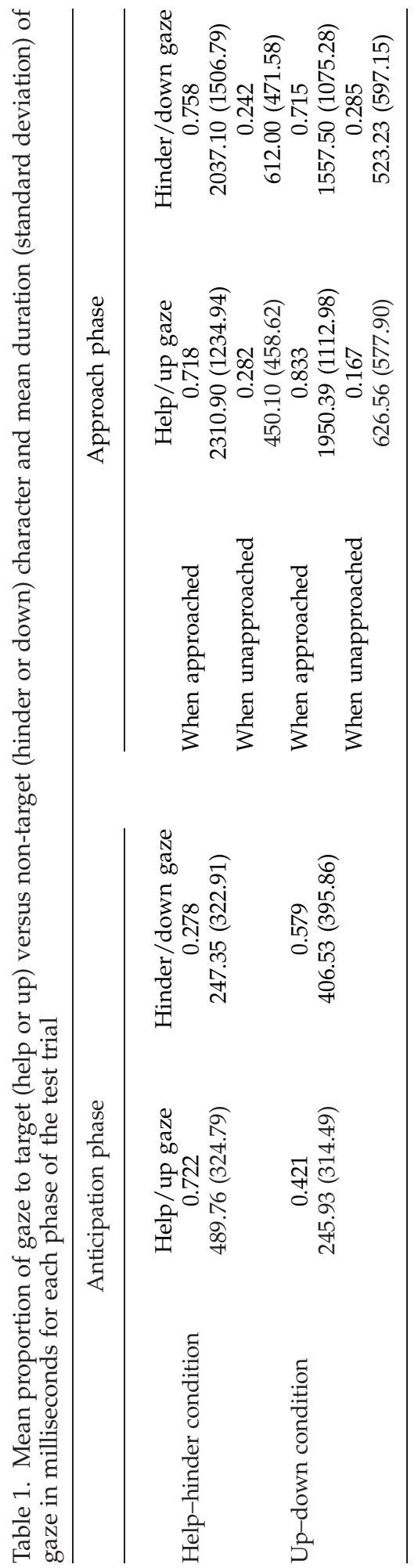




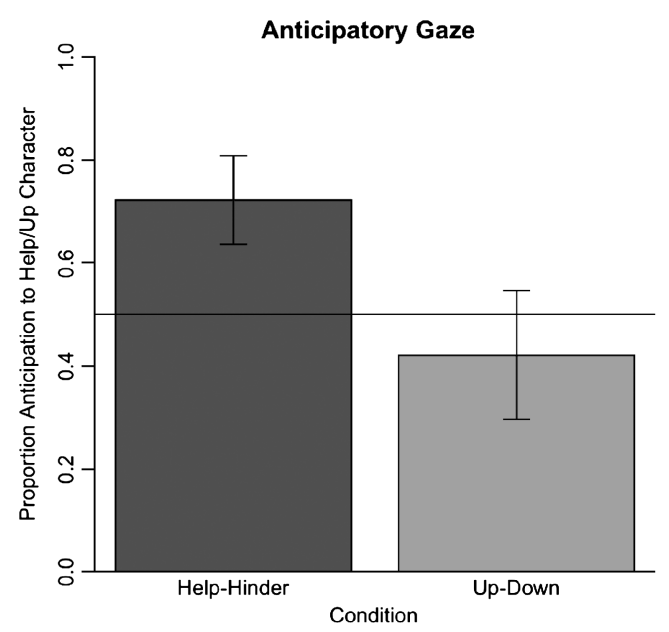

Figure 3. Proportion of anticipatory gaze to the help or up character in each condition.

$p=0.15)$ on infants' gaze to the approached character. There was a marginally significant bias to look at the blue triangle over the yellow square $(B=0.20, t=1.83, p=0.07)$, although this factor of target shape did not interact with any of the other variables (all $p s>0.10$ ). Finally, there was a marginally significant interaction between condition and whether the target character was approached $(B=0.40, t=1.73, p=0.09)$, but follow-up tests revealed no significant simple effects (all $p s>0.10$ ). Importantly, this finding rules out the possibility that infants have an overall bias toward looking at the helper. If infants were biased by their own preference to gaze at the helper, they would continue to show this effect in the approach phase, on top of any effect of whom the agent was approaching.

Together, the results show that infants looked more to the helper in expectation of his approach, whereas in the approach phase, they looked longer to the character the agent was approaching, with no additional effect of who was being approached.

\section{DISCUSSION}

Infants who watch others interact from a third-person perspective can anticipate their behaviour based on social evaluations. Specifically, the current study revealed that infants predict that an agent will approach a character who previously helped him in his goal of climbing up a hill over one who previously hindered him in that goal. In contrast, they have no such expectation for the agent's preferences for characters whose movements were similar to the helper's and hinderer's but did not actually cause the agents' own movement. This second condition shows that infants' predictions in the first condition cannot be explained by lower-level perceptual features of the stimuli (e.g. that upward movement with another animate character is inherently considered positive). Together, the findings reveal that infants attribute social valence only when causal contact is present in a helping versus hindering event.

We would certainly not argue that the only cue for causality that infants can use is physical contact. Infants can interpret causality in temporally contingent actions that do not involve contact, for example when one agent approaches another and 
the second moves away before being contacted (Schlottmann et al., 2009). However, in our study, contact is presented as the cause for the agent's success or failure in reaching his goal. Although some contingency of movement was present in both conditions, in the up-down condition, the agent was already moving, and the contingencies that were present between the movements of the agent and other character (e.g. the short stops and starts) would not be a clear cause for the agent's movement.

Our findings build on previous results using habituation measures to show that infants find the agent's approach of the hinderer to be incongruent (Hamlin \& Wynn, in press; Hamlin et al., 2007a, b; 2010; Kuhlmeier et al., 2003). The measures in these previous studies are limited by their inability to reveal what infants attend to and expect during an interaction. They can only give an idea of infants' interpretation of an event that has already occurred rather than revealing an understanding of ongoing behaviour. Moreover, although 6- and 10-month-old infants' choosing to reach for a particular character (e.g. helper over hinderer) reveals that even younger infants are using the social information they gather from observing others' interactions in an active way (Hamlin et al., 2007a, b), this finding is focused on infants' own behaviour and preferences and thus does not show that infants of this age are able to predict others' behaviours during ongoing interactions.

The current findings are also significant in that we obtained visual anticipation measures from infants' first test trial in a novel scene. Other studies using visual anticipations tend to use data from repeated trials (Falck-Ytter et al., 2006; Gredebäck et al., 2009; Hunnius \& Bekkering, 2010; Paulus et al., 2011) or to train anticipations using discriminative cues (e.g. identical sequences of events across familiarization and test trials: Southgate et al., 2007). For the current study, we chose not to use repeated test trials or training. We felt it was important to assess infants' initial expectation about the agent's behaviour, and there is evidence that infants of this age can anticipate actions based on the context of a situation without previously witnessing the displayed action. For example, Gredebäck and Melinder (2010) and Hunnius and Bekkering (2010) found that 12-month-olds already predicted the goal of an action event the first time they witnessed it. Our findings, along with those of Hunnius and Bekkering (2010), show that at 12 months, approximately half of infants will make spontaneous first trial anticipatory gaze shifts to an action goal, suggesting that action anticipation is still developing at this age.

Could infants simply have been relying on their own preference for the helpful character rather than predicting the agent's behaviour in the novel context? The findings from the approach phase of the current experiment rule out that possibility. That is, if infants' anticipatory gaze was driven by their own preference for the helper, then this preference should be present throughout the trial, even after the agent approached one of the two characters. However, no such effect was found. In addition, all but two of the infants who did not make an anticipation simply tracked the movement of the agent, again failing to provide evidence that they have a bias to gaze at the helper. This provides support for the conclusion that infants' visual anticipations were based on their predictions about the agent's behaviour.

In the current research, we chose to study 12-month-olds because they have been shown to be able to reliably anticipate others' goal-directed actions at that age (e.g. Falck-Ytter et al., 2006; Gredebäck \& Melinder, 2010). In addition, infants at this age are beginning to show spontaneous helpful (e.g. Liszkowski et al., 2006) and empathic (e.g. Zahn-Waxler et al., 1992) behaviour, an indication that they understand, predict and can act on their evaluations of others' behaviour. 
Previous research on social evaluation has been carried out with infants as young as 3 months of age (Hamlin et al., 2010). However, because we were measuring action anticipation, we selected an older age group to begin our investigation. Another difference between the social evaluation studies on very young infants and the current study is the infant's perspective. It has been shown that 3-month-old infants themselves prefer a prosocial agent (Hamlin et al., 2010), whereas only by 10 months do they show third-party understanding that someone else will prefer a prosocial agent (Hamlin et al., 2007). Thus, there could be a transition from first-person preferences to third-party understanding of others' preferences that is also involved in the development of social evaluation (see Moore, 2006). Further research exploring the full trajectory of this development will certainly be insightful.

Being able to predict future actions is an important skill for understanding social interactions from a third-person perspective and for successfully engaging in ongoing interactions. The current study revealed that 12-month-old infants make predictions about others' behaviour and preferences based on social evaluations of those individuals' interactions. By revealing how infants' interpret and predict events, action anticipation can shed new light on cognitive development in many domains.

\section{CONFLICT OF INTEREST}

The authors have declared that there is no conflict of interest.

\section{REFERENCES}

Aslin, R. N. (2007). What's in a look? Developmental Science, 10(1), 48-53.

Cohen, L. B., \& Amsel, G. (1998). Precursors to infants' perception of the causality of a simple event. Infant Behavior \& Development, 21(4), 713-731.

Falck-Ytter, T., Gredebäck, G., \& von Hofsten, C. (2006). Infants predict other people's action goals. Nature Neuroscience, 9(7), 878-879.

Gredebäck, G., Johnson, S., \& Von Hofsten, C. (2010). Eye tracking in infancy research. Developmental Neuropsychology, 35(1), 1-19.

Gredebäck, G., \& Melinder, A. (2010). Infants' understanding of everyday social interactions: A dual process account. Cognition, 114(2), 197-206.

Gredebäck, G., Stasiewicz, D., Falck-Ytter, T., Rosander, K., \& von Hofsten, C. (2009). Action type and goal type modulate goal-directed gaze shifts in 14-month-old infants. Developmental Psychology, 45(4), 1190.

Hamlin, J. K., Wynn, K., \& Bloom, P. (2007). Social evaluation by preverbal infants. Nature, 450(7169), 557-559.

Hamlin, J. K., Wynn, K., \& Bloom, P. (2007b). Social evaluation by preverbal infants. Nature, 450(7169), 557-559.

Hamlin, J. K., Wynn, K., \& Bloom, P. (2010). Three-month-olds show a negativity bias in their social evaluations. Developmental Science, 13(6), 923-929.

Hood, B., Cole-Davies, V., \& Dias, M. (2003). Looking and search measures of object knowledge in preschool children. Developmental Psychology, 39(1), 61-70.

Hunnius, S., \& Bekkering, H. (2010). The early development of object knowledge: A study of infants' visual anticipations during action observation. Developmental Psychology, $46(2), 446$.

Kuhlmeier, V., Wynn, K., \& Bloom, P. (2003). Attribution of dispositional states by 12month-olds. Psychological Science, 14(5), 402-408.

Leslie, A. M. (1984). Spatiotemporal continuity and the perception of causality in infants. Perception, 13(3), 287-305. 
Liszkowski, U., Carpenter, M., Striano, T., \& Tomasello, M. (2006). 12- and 18-month-olds point to provide information for others. Journal of Cognition and Development, 7(2), 173-187.

Moore, C. (2006). Representing intentional relations and acting intentionally in infancy: Current insights and open questions. In G. Knoblich, I. Thornton, M. Grosjean, \& M. Shiffrar, (Eds.), Human body perception from the inside out (pp. 427-442). New York: Oxford University Press.

Oakes, L. M. (1994). Development of infants' use of continuity cues in their perception of causality. Developmental Psychology, 30(6), 869-879.

Paulus, M., Hunnius, S., van Wijngaarden, C., Vrins, S., van Rooij, I., \& Bekkering, H. (2011). The role of frequency information and teleological reasoning in infants' and adults' action prediction. Developmental Psychology, 47(4), 976-983.

Premack, D., \& Premack, A. J. (1997). Infants attribute value to the goal-directed actions of self-propelled objects. Journal of Cognitive Neuroscience, 9(6), 848-856.

Schlottmann, A., Surian, L., \& Ray, E. (2009). Causal perception of action-and-reaction sequences in 8-to 10-month-olds. Journal of Experimental Child Psychology, 103(1), 87-107.

Southgate, V., Senju, A., \& Csibra, G. (2007). Action anticipation through attribution of false belief by 2-year-olds. Psychological Science, 18(7), 587.

Zahn-Waxler, C., Radke-Yarrow, M., Wagner, E., \& Chapman, M. (1992). Development of concern for others. Developmental Psychology, 28(1), 126-136. 